\title{
Efficacy, safety, and impact on hospitalizations of paliperidone palmitate in recent-onset schizophrenia
}

This article was published in the following Dove Press journal:

Neuropsychiatric Disease and Treatment

II March 2015

Number of times this article has been viewed

\author{
Fan Zhang' \\ Tianmei $\mathrm{Si}^{2,3}$ \\ Chiun-Fang Chiou ${ }^{4}$ \\ Anthony WF Harris ${ }^{5}$ \\ Chang Yoon $\mathrm{Kim}^{6}$ \\ Padmashree Jahagirdar ${ }^{7}$ \\ Steve Ascher ${ }^{8}$ \\ 'Xian Janssen Pharmaceuticals, \\ Beijing, People's Republic of \\ China; ${ }^{2}$ Department of Clinical \\ Psychopharmacology, Peking \\ University Institute of Mental \\ Health, Beijing, People's Republic of \\ China; ${ }^{3}$ Key Laboratory of Mental \\ Health, Ministry of Health (Peking \\ University), Beijing, People's Republic \\ of China; ${ }^{4}$ Janssen Asia-Pacific, \\ Singapore; ${ }^{5}$ Discipline of Psychiatry, \\ Sydney Medical School, University of \\ Sydney, Australia and Brain Dynamics \\ Center, Westmead Millennium \\ Institute for Medical Research, \\ Westmead, NSW, Australia; ${ }^{6}$ Asan \\ Medical Center, University of Ulsan \\ College of Medicine, Seoul, South \\ Korea; ${ }^{7}$ Theorem Clinical Research, \\ Bangalore, India; ${ }^{8}$ Janssen Research and \\ Development, LLC, Titusville, NJ, USA
}

Correspondence: Fan Zhang

Xian Janssen Pharmaceuticals,

I4F, Tower 3, China Central Place,

No 77, Jian Guo Road, Chaoyang District,

100025 Beijing, People's Republic of China

Tel +86 I0 582I 8766

Email fzhang21@its.jnj.com
Objective: To evaluate the efficacy, safety, and impact on hospitalizations of long-acting injectable paliperidone palmitate (PP) treatment, in patients with recent-onset schizophrenia who had not responded satisfactorily to oral antipsychotics.

Methods: In this 18-month, open-label, Phase-IIIb study from Asia-Pacific region, patients (18-50 years) with recent-onset ( $\leq 5$ years) schizophrenia unsatisfactorily treated with previous oral antipsychotics were initiated on PP $150 \mathrm{mg}$ eq on day $1,100 \mathrm{mg}$ eq on day 8 , followed by flexible once monthly maintenance doses of $50-150 \mathrm{mg}$ eq. The number and duration of hospitalizations were compared using a mirror analysis method between two periods: retrospective (12 months before PP initiation) and prospective (12 and 18 months after PP treatment) periods.

Results: A total of 303 out of 521 (58\%) patients (mean age, 28.7 years; 65.5\% men, 92.5\% Asian) completed the study. Positive and Negative Syndrome Scale (PANSS) total score improved significantly from baseline to month 18 (mean [standard deviation, SD] change: -11.3 [21.38], $P<0.0001$, primary endpoint). Subgroup analysis revealed greater improvements among patients with worse disease severity at baseline: PANSS $\geq 70$ versus $<70$ (mean [SD] change: -23.1 [24.62] vs -4.7 [15.98], $P<0.0001$ each). Secondary efficacy endpoints such as Clinical Global Impression of Schizophrenia (CGI-SCH), Medication Satisfaction Questionnaire (MSQ) scores showed significant improvements $(P<0.0001)$ from baseline; $33.3 \%$ patients achieved symptom remission. In mirror analyses set $(\mathrm{N}=474)$, PP significantly $(P<0.0001)$ reduced mean number of hospitalization days/person/year (12-month: 74.3 vs 19.7 ; 18 -month: 74.3 vs 18.9 ) as well as percentage of patients requiring hospitalization in past 12 months (12-month: $39.7 \%$ vs $24.6 \%$; 18-month: $39.7 \%$ vs $25 \%$ ), and PP treatment increased the proportion of patients not requiring hospitalization (12-month: $60.3 \%$ vs $75.4 \%$; 18 -month: $60.3 \%$ vs $75 \%$ ) from retrospective to prospective period. Adverse events ( $\geq 15 \%$ ) were extrapyramidal symptoms-related $(31.3 \%)$, injection-site pain (18.6\%), and insomnia (15.2\%).

Conclusion: PP was efficacious and generally tolerable with significant reductions observed in both number of hospitalizations and days spent in hospital.

Trial registration number: ClinicalTrials.gov: NCT01051531.

Keywords: atypical antipsychotics, long-acting injectables, open-label, paliperidone palmitate, schizophrenia

\section{Introduction}

Clinical management of schizophrenia remains a major challenge due to frequent relapses, persistence of psychotic symptoms, nonadherence to antipsychotic medications, recurrent hospitalizations, and increased burden on healthcare resources. ${ }^{1-5}$ Although variable, nonadherence rates among patients with schizophrenia are estimated to range from $40 \%$ to $70 \% .^{6-8}$ 
The causes for poor adherence to antipsychotic treatments are multifactorial: patient-related (poor insight, depression, disorganization, substance abuse), treatment-related (low efficacy, complexity of regimen, adverse events), and lack of support or therapeutic alliance with the treating team. ${ }^{6,8-10}$ Among patients recently diagnosed with schizophrenia, treatment discontinuity along with partial nonadherence ${ }^{11}$ (ie, elapsed time of 1-10 days/year for prescribed antipsychotic therapy resumption) were observed to increase the risk of relapse, ${ }^{12,13}$ and that of hospitalization (odds ratio $=1.98$ ). ${ }^{11}$ Delayed access to mental healthcare in the critical period of the 1st years of illness can result in an incomplete or slow recovery culminating into poor outcome. ${ }^{14,15}$ In addition, the 1st years of illness appear to be a critical period as relapses can cause negative consequences, and permanent deficits are observed even during the initial stages of illness. ${ }^{12,16,17}$ Therefore, early intervention and uninterrupted treatment are important considerations to achieve long-term symptom remission, to prevent illness exacerbation, and reduce the overall burden and disease costs in schizophrenia. ${ }^{12,18}$

Long-acting injectable (LAI) formulations of atypical antipsychotics offer several potential advantages over the oral counterparts, most importantly the ability to reduce relapse as shown in recent head-to-head comparison studies and a meta-analysis. ${ }^{19-21}$ In addition, LAIs allow clinicians to better differentiate nonadherence from nonresponse, lower the risk of accidental or deliberate overdose of medication, and better monitor compliance. LAIs have improved bioavailability and a more predictable correlation between the dose and plasma levels. ${ }^{2,7,22-27}$ Despite these benefits, LAI formulations have been traditionally considered for use after clinical stabilization with oral antipsychotics in the maintenance treatment of patients with schizophrenia. ${ }^{28}$ Most often, they have been reserved as a "last resort" for use in patients who have severely deteriorated or frequently relapsed, and only after the failure of orally administered pharmacotherapies. ${ }^{22,29}$ Paliperidone palmitate (PP), is a once-monthly LAI atypical antipsychotic, approved in the US, Europe, and all major Asia-Pacific countries for acute and maintenance treatment of schizophrenia. Compelling evidence supports PP's effectiveness and safety $1,17,30-40$ - including in Asian patients with schizophrenia. ${ }^{41,42}$

The aim of this study was to assess the effectiveness of switching from oral antipsychotics to PP in a Phase-IIIb, long-term (18 months), open-label study by evaluating treatment response, safety, and impact on hospitalization in patients with recent-onset schizophrenia from the AsiaPacific region.

\section{Methods}

\section{Patients}

All patients were aged between 18 and 50 years (inclusive) with a diagnosis ( $\leq 5$ years before screening) of schizophrenia (as per the Diagnostic and Statistical Manual of Mental Disorders, 4th Edition [DSM-IV] criteria), who had not responded adequately to previous oral antipsychotics. Previous antipsychotic treatment was considered unsuccessful due to one or more of the following reasons: lack of efficacy, lack of tolerability or safety, or lack of compliance.

Major exclusion criteria included psychiatric diagnosis due to medication/substance abuse, or a general medical condition; treatment-resistance; $D S M-I V$ diagnosis of substance dependence within 6 months before study entry; treatment with an LAI antipsychotic within three injection cycles before baseline, or clozapine within 3 months before screening; history of neuroleptic malignant syndrome, or tardive dyskinesia; women not using an acceptable method of contraception unless postmenopausal for $>1$ year, surgically sterile, or abstinent.

Independent Ethics Committee or Institutional Review Board at each study site approved the protocol and the study was conducted in accordance with the ethical principles that have their origin in the Declaration of Helsinki and that are consistent with Good Clinical Practices and applicable regulatory requirements. All patients or their legally acceptable representatives provided written informed consent before entering the study (NCT01051531).

\section{Study design and treatment}

This 18-month, nonrandomized, single-arm, open-label, multicenter study was conducted between April 2010 and May 2013 across nine countries of the Asia-Pacific region including Australia, People's Republic of China, Hong Kong, Korea, Malaysia, New Zealand, Philippines, Taiwan, and Thailand. The study consisted of an up to 7-day screening phase including washout of disallowed medications, followed by an 18-month open-label treatment phase, and an end-of-study/ early-withdrawal visit a month after the last PP injection.

\section{Dosage and administration}

Eligible patients were switched to PP from their existing oral antipsychotic medication due to either efficacy or tolerability issues. Patients were required to discontinue previous oral antipsychotic medications before day 1 ; those pretreated with high doses of antipsychotics that had sedating and/or anticholinergic properties were allowed to taper off these medications through the 1st month of the study. Treatment with PP 
was initiated at a dose of $150 \mathrm{mg}$ eq on day $1,100 \mathrm{mg}$ eq on day 8 (both doses in the deltoid muscle), followed by flexible once-monthly injections of $50,75,100$, or $150 \mathrm{mg}$ eq in the deltoid or gluteal muscle at investigator's discretion.

\section{Concomitant medications}

Psychotropic medications such as mood stabilizers, antidepressants (except any oral or injectable antipsychotic other than PP); lorazepam $0.5 \mathrm{mg}$ ( $\leq 6 \mathrm{mg}$ /day orally) for not more than 10 days in a month as needed; benzodiazepines if they had been used at a stable dose for $\geq 3$ months before screening; and anticholinergic medication up to 8 weeks from day 1 then tapered off at investigator's discretion for treating movement disorders, were allowed.

\section{Study assessments Efficacy}

The primary efficacy endpoint was the change in Positive and Negative Syndrome Scale (PANSS) total score from baseline to endpoint (month 18). Exploratory subgroup analysis was performed for PANSS baseline total score categories $(\geq 70$ and $<70$ ). Major secondary efficacy endpoints included changes from baseline to month 18 in PANSS subdomains/ symptom factor scores, Clinical Global Impression of Schizophrenia (CGI-SCH), Medication Satisfaction Questionnaires (MSQ) scores, and symptom remission, defined as absent to mild core symptoms for 6 or more months on the following PANSS items: P1 Delusions, P2 Conceptual disorganization, P3 Hallucinatory behavior, N1 Blunted affect, N4 Passive/apathetic social withdrawal, N6 Lack of spontaneity and conversation flow, G5 Mannerisms and posturing, and G9 Unusual thought content. ${ }^{43}$

For PANSS baseline score $<70$ group, changes in PANSS total score, CGI-SCH overall severity score and MSQ score were analyzed at month 18 to check for further improvement/ worsening in this subgroup. Response rate, ie, percentage of patients achieving responses of a magnitude of $\geq 30 \%$ for PANSS total score was also evaluated. Response criteria was defined as percent reduction in PANSS total score from baseline $=($ postbaseline value - baseline value $) \times 100 /$ (baseline value -30), 30 being the lowest possible value for PANSS total score. Furthermore, the number and percentage of patients reporting an improvement ( $\geq 20 \%$ decrease) or worsening ( $\geq 20 \%$ increase) in PANSS total score from baseline were calculated at each scheduled assessment. In addition, an exploratory subgroup analysis for baseline CGI-SCH overall severity score was performed, ie, $\geq 4$ and $<4$ categories.
Treatment adherence percentage was calculated as (number of actual doses/number of planned doses) $\times 100$ for patients who had at least $70 \%$ treatment adherence during the study. All clinicians who administered these scales had passed a qualification examination prior to monitoring patients in the study.

\section{Healthcare resource utilization}

Healthcare resource utilization data captured hospitalizations including at least one overnight stay due to psychiatric reasons. These data were collected to assess patients' utilization of healthcare services for two periods: retrospective period (12 months before PP initiation) and at every 3-month interval during the prospective period (after PP treatment). The start and end dates for hospitalizations were recorded. Protocol-mandated procedures, tests, and encounters were excluded.

\section{Safety}

Safety assessments included recording and monitoring of treatment-emergent adverse events (TEAEs), clinical laboratory tests, vital sign measurements, physical examinations, movement disorder evaluation using Extrapyramidal Symptom Rating Scale-Abbreviated (ESRS-A) scale, and Clinical Global Impression of Movement Severity (CGI-MS) scale.

\section{Statistical analyses}

\section{Sample size determination}

Under the assumption of a 6-point reduction in PANSS total score (a minimum clinically relevant difference) before switching to PP, we tested for an additional 3-point improvement. Based on prior studies of LAI, an average 3-unit additional reduction from baseline in the PANSS total score could be expected 18 months after switching to PP from prior oral antipsychotics in stable patients. ${ }^{39,44}$

A sample size of 469 patients was needed to detect a difference of 3 units (for the null hypothesis of $\geq-3$ change) in the PANSS total score with a standard deviation (SD) of 20 at $90 \%$ power and a significance level of 0.025 using onesided, one-sample $t$-test. Assuming a 20\% dropout rate, 587 patients were to be recruited.

\section{Primary efficacy endpoint analyses}

Primary efficacy parameter was analyzed using a one-sample $t$-test and one-sided 95\% confidence intervals (CIs). Efficacy of PP was concluded if upper limit of one-sided 95\% CI for the change from baseline in PANSS total score (at month 18 using last observation carried forward [LOCF]) was less than 
-3 points. Exploratory subgroup analyses for PANSS baseline total score categories ( $\geq 70$ and $<70$ ) was performed using analysis of covariance and the results were summarized.

\section{Secondary efficacy endpoint analyses}

Secondary efficacy parameters (PANSS total score and PANSS subdomains factors, CGI-SCH, MSQ scale scores) were evaluated using a paired $t$-test. ANOVA (analysis of variance) model was used for subgroup analysis of baseline CGI-SCH overall severity score categories. Symptom remission was assessed using McNemar's test. LOCF approach was used for missing data imputation, with no adjustment made for multiplicity.

\section{Safety and hospitalizations}

Safety results and hospital visits were descriptively summarized. The number and percent of patients hospitalized and days spent in hospital were summarized along with two-sided 95\% CIs, and paired $t$-test was used to detect within-patient change in per-person years from a retrospective period of 12 months compared with the prospective study period.

Hospitalizations were compared using the "mirror analysis" method. The mean number and percent of patients hospitalized as well as duration of hospital stays (expressed as per-person/year) for 12 months before starting PP (retrospective period) were compared with 12 months after PP initiation and at the end of PP treatment (18-month) in the prospective period. Additionally, a sensitivity analysis after excluding patients with schizophrenia history of $<1$ year was conducted for the mean number of hospital days/person/year of patients in the mirror analyses population.

\section{Analyses populations}

All efficacy analyses were performed on the intent-to-treat (ITT) population, which included all patients who received at least one injection of PP. This population also represents safety population. Healthcare utilization population comprised of all ITT patients who participated in healthcare utilization data collection. Mirror analyses population consisted of all healthcare utilization patients with retrospective data (including hospitalizations visits) available for the 12 months prior to day 1 .

\section{Results}

\section{Patient disposition and clinical characteristics}

A total of 510 out of 585 enrolled patients (87.2\%) were from Asian countries: People's Republic of China (20.2\%),
Malaysia (20.2\%), Korea (17.6\%), Taiwan (11.3\%), Thailand (9.6\%), Philippines (5.1\%), and Hong Kong (3.2\%), and the remainder $75(12.8 \%)$ patients were all from Australia and New Zealand. The ITT and safety populations comprised 521 patients, 474 of whom were evaluable for healthcare resource utilization and also constituted the mirror analyses population. Overall, 303 of $521(58.2 \%)$ patients from the ITT population completed the study. The most common reasons for discontinuations (ITT population) from the study were: withdrawal of consent $(n=67,12.9 \%)$, lack of efficacy $(n=50,9.6 \%)$, and TEAEs $(n=42,8.1 \%)$. One of the sites in Australia that enrolled 25 patients was noncompliant with GCP. The data from this site were excluded from the efficacy and safety analysis. The baseline data (excluding this site) are presented.

Patients were mostly men (65.5\%) and had a median age of 27 years (range: 18-56 years) (Table 1). Most patients $(68.9 \%)$ were diagnosed with schizophrenia within the past 3 years. Previous oral antipsychotic as well as concomitant (those tapering-off their previous medications in the $1 \mathrm{st}$ month) medications were: risperidone (24\% and $28.4 \%)$, paliperidone (16.3\% and $13.2 \%)$, haloperidol $(8.3 \%$ and $5.8 \%)$, and olanzapine $(6.9 \%$ and $10.7 \%)$.

\section{Treatment adherence and extent of exposure}

Overall, 93.7\% patients were $\geq 70 \%$ treatment adherent, and the mean (SD) treatment adherence was 95.7\% (12.28) (range: 33\%-120\%). A mean (SD) 13.9 (6.78) doses were administered over 382.6 (202.07) days. The mean (SD) dose of PP was 108.1 (24.23) mg eq (95\% CI: 106.01; 110.18), and the mean (SD) maintenance dose per PP injection (excluding initiation regimen) after day $8(\mathrm{n}=471)$ was 100.8 (28.33) mg eq (95\% CI: 98.27; 103.40).

Patients $(n=471,65.6 \%)$ were mostly on a fixed maintenance dose of $75 \mathrm{mg}$ eq on day 38, while some patients required higher dose levels (100 mg eq: $25.1 \%$, $150 \mathrm{mg}$ eq: $9.1 \%$, permitted per protocol amendment). Only one patient required a dose reduction to $50 \mathrm{mg}$ eq on day 38 . Overall, 162 patients required dose adjustments from day 38 onward because the fixed dose of $75 \mathrm{mg}$ eq in the original protocol led to insufficient efficacy in the majority (98.1\%) of the patients, and tolerability concerns in $1.2 \%$.

\section{Efficacy findings}

Primary efficacy parameters

There was a significant $(P<0.0001)$ and clinically meaningful improvement in mean (SD) PANSS total score of -11.3 
Table I Baseline demographics and clinical characteristics of patients recently diagnosed with schizophrenia (intent-to-treat population)

\begin{tabular}{|c|c|}
\hline Parameters & $\begin{array}{l}\text { Paliperidone } \\
\text { palmitate }(\mathrm{N}=52 \mathrm{l})\end{array}$ \\
\hline Age (years), mean (SD) & $28.7(7.95)$ \\
\hline Weight ${ }^{\mathrm{a}}(\mathrm{kg})$, mean (SD) & $68.4(16.82)$ \\
\hline \multicolumn{2}{|l|}{ Sex, $n(\%)$} \\
\hline Men & $34 \mid(65.5)$ \\
\hline Women & $180(34.5)$ \\
\hline \multicolumn{2}{|l|}{ Race, n (\%) } \\
\hline Asian & $482(92.5)$ \\
\hline White & $31(6.0)$ \\
\hline African American & $3(0.6)$ \\
\hline Native Hawaiian or other Pacific Islander & $\mathrm{I}(0.2)$ \\
\hline Other & $4(0.8)$ \\
\hline \multicolumn{2}{|l|}{ Schizophrenia type, n (\%) } \\
\hline Paranoid & $387(74.3)$ \\
\hline Undifferentiated & $86(16.5)$ \\
\hline Disorganized & $28(5.4)$ \\
\hline Residual & $20(3.8)$ \\
\hline \multicolumn{2}{|c|}{ Elapsed time since first diagnosis by yearly interval, $n$ (\%) } \\
\hline 0 to $<1$ & $191(36.7)$ \\
\hline I to $<2$ & $82(15.7)$ \\
\hline 2 to $<3$ & $86(16.5)$ \\
\hline 3 to $<4$ & $89(17.1)$ \\
\hline$\geq 4$ & $73(14.0)$ \\
\hline Prior antipsychotic use, n (\%) & $323(62.0)$ \\
\hline PANSS baseline total score, ${ }^{a}$ mean (SD) & $64.1(19.09)$ \\
\hline \multicolumn{2}{|l|}{ PANSS baseline total score categories, ${ }^{\text {a }}$ (\%) } \\
\hline$<70$ & $332(63.7)$ \\
\hline$\geq 70$ & $188(36.1)$ \\
\hline \multicolumn{2}{|l|}{ CGI-SCH baseline overall severity score, ${ }^{a} \mathrm{n}(\%)$} \\
\hline$<4$ & $283(54.3)$ \\
\hline$\geq 4$ & $237(45.5)$ \\
\hline \multicolumn{2}{|c|}{ Prior psychiatric hospitalizations ( 2 years before baseline), n (\%) } \\
\hline None & $334(64.1)$ \\
\hline At least once & $187(35.9)$ \\
\hline
\end{tabular}

Note: ${ }^{\mathrm{a}} \mathrm{n}=520$.

Abbreviations: CGI-SCH, Clinical Global Impression of Schizophrenia; PANSS, Positive and Negative Syndrome Scale; SD, standard deviation.

(21.38) from baseline to endpoint (one-sided 95\% CI: [-infinity; -9.7]) (Figure 1). A subgroup analyses of PANSS baseline total score categories (Figure 2) revealed that the improvement was significantly $(P<0.0001)$ more pronounced among patients with worse disease severity at baseline: PANSS baseline total score $\geq 70$ compared with $<70$ (mean [SD] change: -23.1 [24.62] vs -4.7 [15.98])

\section{Secondary efficacy parameters}

There were significant $(P<0.0001)$ improvements in all five symptom domains of PANSS (Table 2). Other secondary efficacy parameters including total CGI-SCH, all CGI-SCH

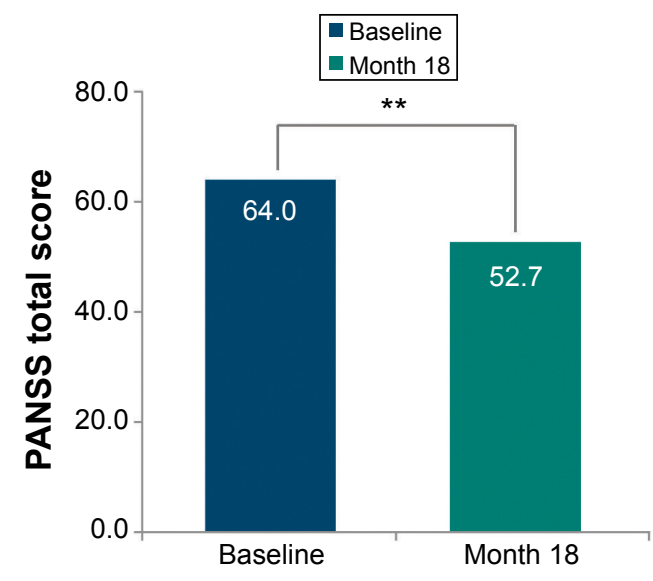

Figure I Change from baseline to month 18 in primary efficacy parameter (intentto-treat population; LOCF).

Notes: **Compared with baseline, $P<0.0001$. $P$-value is from one-sided paired $t$-test for the null hypothesis that change is $\geq-3$.

Abbreviations: LOCF, last observation carried forward; PANSS, Positive and Negative Syndrome Scale.

subindices, and MSQ scores also showed significant $(P<0.0001)$ improvements with PP treatment. Subgroup analysis findings for baseline CGI-SCH overall severity score categories suggested significantly greater improvements for patients with worse disease severity at baseline ie, CGI-SCH overall severity score of $\geq 4$ than $<4$ (mean change: -18.2 vs $-5.5, P<0.0001)$. According to subgroup analysis performed to confirm improvement/worsening in PANSS baseline score $<70$ category, majority of patients $(>70 \%)$ had shown improvement/no change in PANSS total score (70.2\%), CGI-SCH overall severity score (84.9\%), and MSQ score (75.9\%) by month 18 (Table 3).

The percentage of responders $(\geq 30 \%$ reduction in PANSS total score) increased at each visit: $35.5 \%$ (day 38), $66.8 \%$ (day 188), $70.2 \%$ (day 368 ), and $73.9 \%$ (day 548); while patients having PANSS reduction between $90 \%$ and $\leq 100 \%$ also increased with subsequent visits: $4.8 \%$ (day 38 ), $12.3 \%$ (day 188), $17.0 \%$ (day 368), and 21.6\% (day 548). As the treatment duration increased, such trends of greater improvement over time were also evident among patients with response rates ranging from $60 \%$ to $70 \%, 70 \%$ to $80 \%$, and $80 \%$ to $90 \%$ (data not shown).

In addition, $45.3 \%$ patients demonstrated an improvement ( $\geq 20 \%$ decrease) in PANSS total score from baseline to endpoint, while the majority (44.1\%) among the remaining patients had a stable condition at each visit. The condition fluctuated or worsened for the remainder of patients (range: $6.1 \%-11.6 \%$ ) across the visits. Notably, 18-month treatment with PP resulted in symptom remission for $33.3 \%$ patients at the end of study, and the results were significant $(P<0.0001)$ from month 6 . 


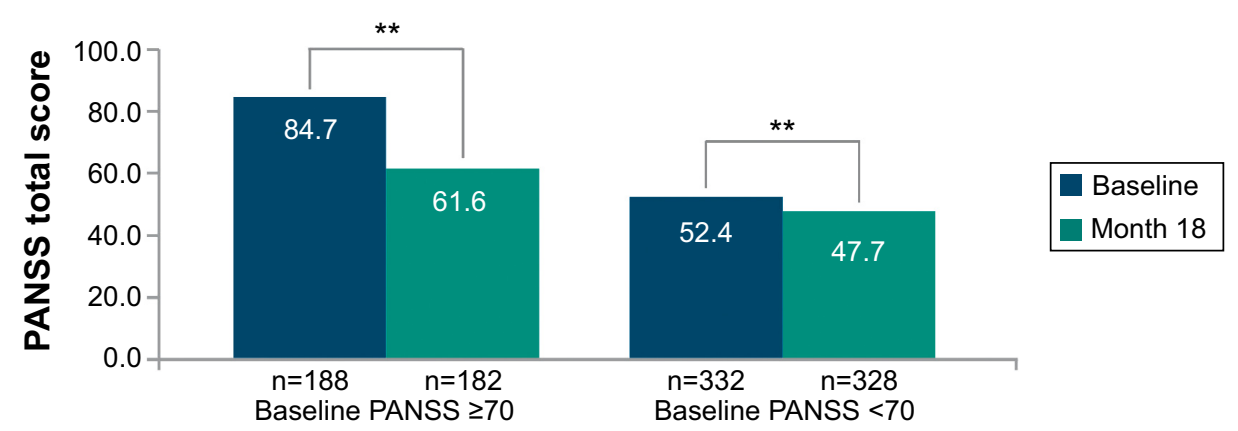

Figure 2 Change from baseline to month 18 in PANSS total score by PANSS baseline total score categories (intent-to-treat population; LOCF) Notes: $* *$ Compared with baseline, $P<0.000$ I. $P$-value is from one-sided paired $t$-test for the null hypothesis that change is $\geq-3$.

Abbreviations: LOCF, last observation carried forward; PANSS, Positive and Negative Syndrome Scale.

\section{Healthcare resource utilization}

Overall, 46 (8.8\%) patients had been hospitalized (at least one overnight stay) for psychiatric reasons; nonetheless, most (7.7\%) were hospitalized only once. The mean (95\% CI) number of hospitalization was $1.2(1.0 ; 1.3)$ and the length of hospital stay was $36.6(22.3 ; 50.9)$ days for the prospective period. During the course of study, $15.4 \%(n=73 / 474)$ patients started PP treatment in the hospital setting.

Over the prospective period ( $\mathrm{N}=474$, mirror analyses set), PP was associated with significantly lower mean number of hospitalization days/person/year versus retrospective period (12-month: 19.77 vs $74.25, P<0.0001 ; 18$-month: 18.93 vs $74.25, P<0.0001$ ) (Figure 3 A). Sensitivity analyses confirmed significant reductions in the mean number of hospitalization days/person/year during prospective versus retrospective period among patients with $>1$ year schizophrenia history (12-month: 20.29 vs $63.31, P<0.0001$; 18 -month: 19.16 vs $63.31, P<0.0001$ ) (Figure $3 \mathrm{~B}$ ), and in those hospitalized in past 12 months (12-month: 36.5 vs 187.01 , $P<0.0001$; 18-month: 34.48 vs 187.01, $P<0.0001$ ) (Figure $3 C)$. In previously hospitalized population, the reductions in the mean number of hospitalization days/person/year were also significant after excluding $<1$ year schizophrenia history patients (12-month: 48.35 vs 205.25, $P<0.0001$; 18 -month: 44.67 vs 205.25, $P<0.0001$ (Figure 3D).

After treatment with PP, compared with the retrospective period, the percentage of patients who required hospitalization significantly reduced (12-month: 39.7\% vs $24.6 \%$; 18 -month: $39.7 \%$ vs $25 \%, P<0.001$ each), and those not requiring hospitalization significantly increased (12-month: $60.3 \%$ vs $75.4 \%$; 18 -month: $60.3 \%$ vs $75 \%, P<0.001$ each). Similarly, sensitivity analyses results showed that in the group of patients with $>1$ year schizophrenia history, there was a significant reduction in the number requiring hospitalization compared to the retrospective period (12-month: $30.8 \%$ vs $23.9 \%$; 18-month: $30.8 \%$ vs $24.5 \%, P<0.001$ each), while those not requiring hospitalization significantly increased (12-month: $69.2 \%$ vs $76.1 \%$; 18 -month: $69.2 \%$ vs $75.5 \%, P<0.001$ each) during prospective period.

\section{Safety and tolerability findings}

Of the enrolled patients, 429 patients $(82.3 \%, \mathrm{n}=521$ safety population) experienced at least one TEAE during the 18-month treatment with PP. Most commonly reported TEAEs (in $\geq 10 \%$ patients) were injection site pain (18.6\%), insomnia (15.2\%), akathisia (13.4\%), and headache (11.3\%) (Table 4). The intensity of these events was mostly mild to moderate. Importantly, the proportion of patients with injection site pain decreased prominently from $17.1 \%$ during the 1 st week of treatment to $1.3 \%$ at the end of 1 month treatment. Two deaths (suicide and pulmonary embolism, $\mathrm{n}=1$ each) occurred during the study. Overall, 76 patients (14.6\%) experienced serious TEAEs, which were mostly related to psychiatric disorders; worsening of schizophrenia symptoms (5.2\%) and psychotic disorder (3.6\%). In all, $66(12.7 \%)$ patients discontinued the study due to TEAEs, psychiatric disorders (7.3\%) being one of the main causes for premature termination.

\section{Safety events of clinical interest}

Extrapyramidal symptoms (EPS) related events were reported by 163 (31\%) patients during the entire study, mostly nonserious (mild to moderate) in intensity. The specific EPS observed in $\geq 2 \%$ patients were akathisia (13.4\%), tremor $(6.3 \%)$, restlessness $(5 \%)$, muscle rigidity $(3.1 \%)$, dystonia and musculoskeletal stiffness (2.5\% each), and Parkinsonism (2.1\%). In total, $7.7 \%$ patients experienced EPS-related TEAEs on maintenance dose level of $150 \mathrm{mg}$ eq. No event of akathisia was serious and only $3(0.6 \%)$ patients 
Table 2 Changes from baseline to month 18 in secondary efficacy parameters (intent-to-treat population; LOCF)

\begin{tabular}{|c|c|}
\hline Secondary efficacy parameters & $\begin{array}{l}\text { Paliperidone palmitate } \\
(\mathrm{N}=52 \mathrm{I})\end{array}$ \\
\hline \multicolumn{2}{|l|}{ PANSS factor scores, ${ }^{\mathrm{a}}$ mean (SD) } \\
\hline \multicolumn{2}{|l|}{ Positive symptoms } \\
\hline Baseline & $18.7(6.94)$ \\
\hline Change from baseline & $-3.8(7.65)^{*}$ \\
\hline \multicolumn{2}{|l|}{ Negative symptoms } \\
\hline Baseline & $16.9(7.01)$ \\
\hline Change from baseline & $-3.0(6.19)^{*}$ \\
\hline \multicolumn{2}{|l|}{ Disorganized thoughts } \\
\hline Baseline & $14.0(4.86)$ \\
\hline Change from baseline & $-2.2(5.30)^{*}$ \\
\hline \multicolumn{2}{|l|}{ Uncontrolled hostility/excitement } \\
\hline Baseline & $6.7(3.43)$ \\
\hline Change from baseline & $-1.1(3.69)^{*}$ \\
\hline \multicolumn{2}{|l|}{ Anxiety/depression } \\
\hline Baseline & $7.7(3.13)$ \\
\hline Change from baseline & $-1.2(3.45)^{*}$ \\
\hline \multicolumn{2}{|l|}{ CGI-SCH score, ${ }^{\mathrm{b}}$ mean (SD) } \\
\hline \multicolumn{2}{|l|}{ Positive symptoms } \\
\hline Baseline & $3.1(1.40)$ \\
\hline Change from baseline & $-0.8(1.57)^{*}$ \\
\hline \multicolumn{2}{|l|}{ Negative symptoms } \\
\hline Baseline & $3.0(1.23)$ \\
\hline Change from baseline & $-0.6(1.14)^{*}$ \\
\hline \multicolumn{2}{|l|}{ Depressive symptoms } \\
\hline Baseline & $1.9(0.99)$ \\
\hline Change from baseline & $-0.3(1.08)^{*}$ \\
\hline \multicolumn{2}{|l|}{ Cognitive symptoms } \\
\hline Baseline & $2.6(1.13)$ \\
\hline Change from baseline & $-0.4(1.19)^{*}$ \\
\hline \multicolumn{2}{|l|}{ Overall severity } \\
\hline Baseline & $3.4(1.10)$ \\
\hline Change from baseline & $-0.8(1.35)^{*}$ \\
\hline \multicolumn{2}{|l|}{ MSQ scores, ${ }^{c}$ mean (SD) } \\
\hline Baseline & $4.3(1.31)$ \\
\hline Change from baseline & $0.8(1.90)^{*}$ \\
\hline
\end{tabular}

Notes: A reduction in PANSS and CGI-SCH scores from baseline represents improvement. An increase in MSQ score from baseline indicates improvement. ${ }^{a} \mathrm{n}=510,{ }^{b} \mathrm{n}=517,{ }^{\mathrm{c}} \mathrm{n}=515, * P<0.0001$.

Abbreviations: CGI-SCH, Clinical Global Impression of Schizophrenia; LOCF, last observation carried forward; MSQ, Medication Satisfaction Questionnaire; PANSS, Positive and Negative Syndrome Scale; SD, standard deviation.

discontinued treatment prematurely because of akathisia. EPS were more common in patients from People's Republic of China (41.7\%), and Philippines (35.7\%).

Prolactin-related TEAEs occurred in 62 (11.9\%) patients, more commonly in women $(25.6 \%, \mathrm{n}=46 / 180)$ than in men $(4.7 \%, n=16 / 341)$. Potential prolactin-related TEAEs such as amenorrhea (11.1\%), menstrual disorder (5.6\%), and hyperprolactinemia (4.4\%) were frequent $(\geq 2 \%)$ among women; sexual dysfunction (2.1\%) was frequent among men. The rate of glucose-related TEAEs was low $(0.6 \%)$ : two reports of diabetes mellitus, one related to inadequate
Table 3 Improvement/worsening in PANSS total score, CGI-SCH overall severity score, and MSQ score at month 18 for baseline PANSS score $<70$ subgroup (intent-to-treat population)

\begin{tabular}{lll}
\hline & $\mathbf{N}(\%)$ & Mean score \\
\hline PANSS total score $(\mathrm{N}=328)$ & & \\
Improved & $219(66.8)$ & 40.0 \\
No change & II (3.4) & 47.9 \\
Worsened & $98(29.9)$ & 64.9 \\
Overall CGI-SCH severity score (N=33I) & \\
Improved & I76 (53.2) & 1.7 \\
No change & I05 (3I.7) & 2.6 \\
Worsened & $50(15.1)$ & 4.0 \\
MSQ score (N=330) & & \\
Improved & I72 (52.0) & 6.0 \\
No change & $79(23.9)$ & 4.9 \\
Worsened & $79(23.9)$ & 3.5 \\
\hline
\end{tabular}

Abbreviations: CGI-SCH, Clinical Global Impression of Schizophrenia; MSQ, Medication Satisfaction Questionnaire; PANSS, Positive and Negative Syndrome Scale.

control of diabetes mellitus and one report of abnormal blood glucose. Weight gain represented an important TEAE. There was a mean increase of $3.91(6.391) \mathrm{kg}(95 \%$ CI: $2.9 ; 4.9)$ in body weight from baseline to end of treatment $(P<0.0001)$. $\mathrm{A} \geq 7 \%$ increase in weight from baseline was reported in $41.8 \%$ patients by the end of treatment. Clinically relevant changes in vital signs (blood pressure and heart rate) were unusual and did not pose any safety concerns. Palpitations or sinus arrhythmia $(0.8 \%$ each), sinus bradycardia, hypertension and orthostatic hypotension $(0.4 \%$ each $)$, and increased blood pressure $(0.2 \%)$ were observed. Significant reductions in CGI-MS scores indicated improvement for Parkinsonism: mean (SD) reduction from baseline in CGI-MS was -0.1 (0.80) $(P<0.0001)$; and for akathisia: mean $(\mathrm{SD})$ reduction was $-0.1(0.77)(P=0.0435)$.

\section{Discussion}

The current study is one of the largest and longest (18 months) studies conducted in the Asia-Pacific region that evaluated the efficacy, safety, and effect on hospital admissions after switching to PP among patients who had failed on their previous oral antipsychotics in recently diagnosed schizophrenia.

Both primary and secondary efficacy results demonstrated that PP treatment resulted in clinically important symptom reductions for patients with a recent diagnosis of schizophrenia, as shown by significant $(P<0.0001)$ improvements in psychotic symptoms as measured by PANSS, overall clinical status (CGI-SCH), and greater patient satisfaction associated with LAI treatment (MSQ). In a subgroup of patients categorized by disease severity at baseline (PANSS baseline total scores $\geq 70$ and $<70$ ), significantly greater improvements 
A

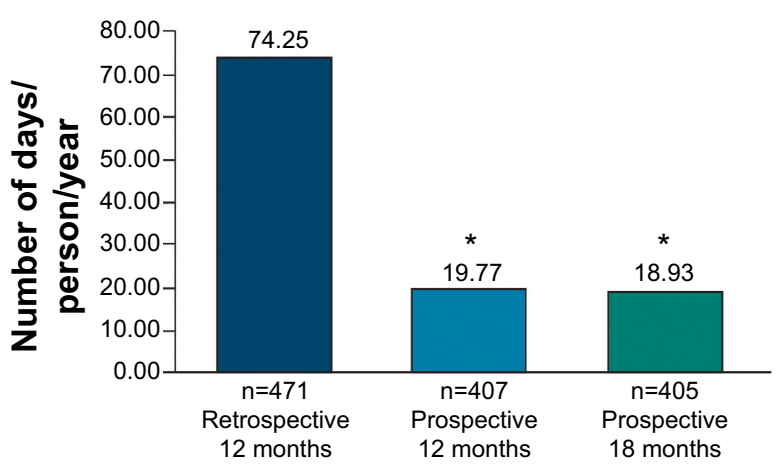

B

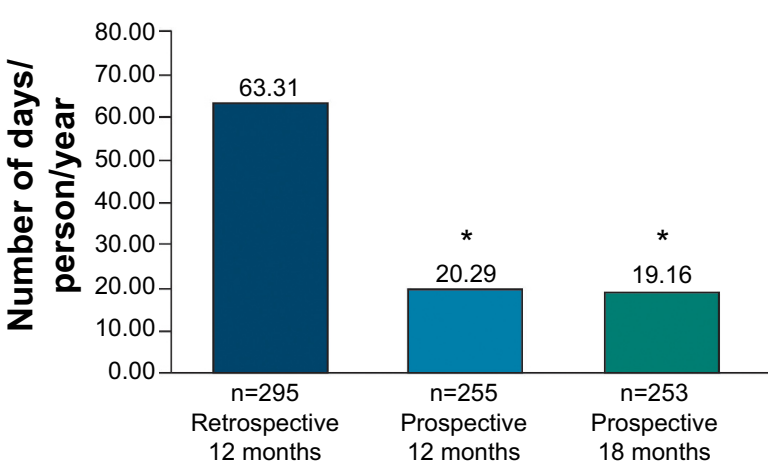

C

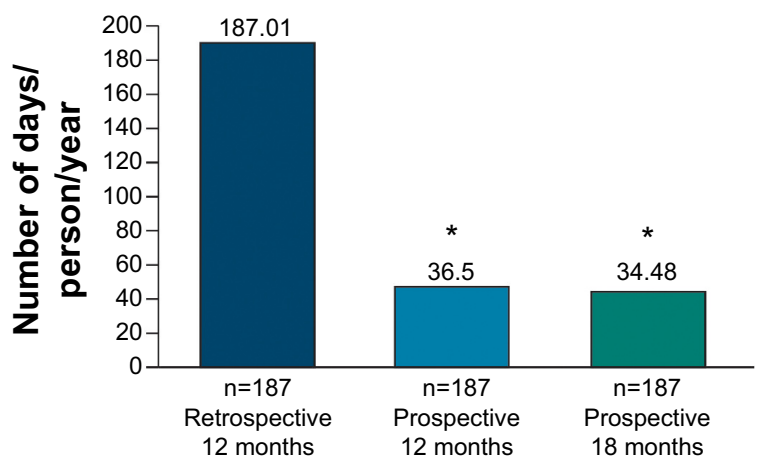

D

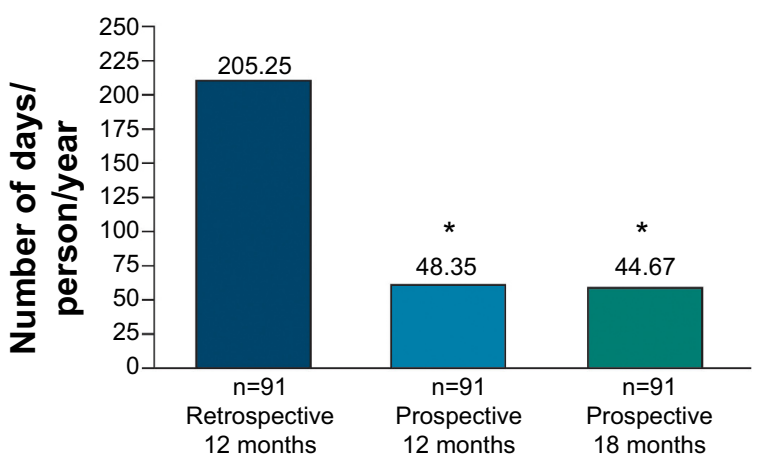

Figure 3 Hospitalizations during retrospective and prospective periods (mirror analyses population).

Notes: (A) Mean number of hospitalization days/person/year for total mirror analyses population. (B) Mean number of hospitalization days/person/year in patients with $>$ I year schizophrenia history. $* \mathrm{P}<0.000 \mathrm{I}$ compared with retrospective period. (C) Mean number of hospitalization days/person/year for patients hospitalized within past 12 months. (D) Mean number of hospitalization days/person/year after excluding $<$ I year schizophrenia history patients. $* P<0.000 \mathrm{I}$.

in symptoms were evident among patients who had worse disease intensity ( $\geq 70$ group) than those with mild disease intensity ( $<70$ group). The latter group remained rather stable across the study period. Pertinent to this, overall approximately $45.3 \%$ patients had improved ( $\geq 20 \%$ decrease in PANSS total score) after PP treatment, while $44.1 \%$ achieved symptom stability at each visit. Overall efficacy results demonstrate robust benefits from PP in patients at the early stages of the disease. Moreover, the pattern of efficacy seen in our study expands evidence from earlier clinical studies of PP, ${ }^{37,39,45,46}$ and supports improved outcomes associated with PP within this subgroup of patients.

The protocol originally required a fixed maintenance dose of $75 \mathrm{mg}$ eq at the third injection, therefore a majority of patients $(65.6 \%)$ were on this dose by day 38 . However, this fixed dose was inadequate in improving schizophrenia symptoms in many patients, which led to the protocol amendment to allow flexible dosing (50-150 mg eq) from third injection (day 38) onward to permit optimization of treatment response in the enrolled patients. The resulting mean dose of $100.8 \mathrm{mg}$ eq was required to maintain the clinical response in patients with early onset of schizophrenia.
There is evidence that treatment of first-episode patients with an LAI atypical antipsychotic increases treatment acceptability, adherence, ${ }^{47}$ and the rate of sustained remission. ${ }^{16}$ It is interesting to note that a high proportion of patients (93.7\%) in the current study demonstrated more than $70 \%$ treatment adherence. Notably, the higher treatment retention rate observed in our study contrasted with the lower adherence rate in a similar study with another LAI (81.8\%), albeit an agent with a fortnightly injection and from a European cultural context. ${ }^{48}$ Of particular interest is the finding that symptom remission was seen in $33.3 \%$ patients in our study. As remission is a critical marker of success in the management of schizophrenia, the results highlight the clinical value of initiating PP monthly injections early in the course of treatment for patients who have not achieved adequate symptom control with oral antipsychotics.

There is a large body of literature that has found a favorable effect of atypical LAIs on the rate of hospitalization. Typically, these use a "before" and "after" study design in which each patient acts as their own control. ${ }^{40,48-56} \mathrm{~A}$ recent meta-analysis on relapse prevention in schizophrenia with LAI versus oral antipsychotics based on randomized 
Table 4 Treatment-emergent adverse events in at least $5 \%$ patients (safety population)

\begin{tabular}{ll}
\hline Most common TEAEs & Paliperidone \\
& palmitate $(\mathbf{N}=\mathbf{5 2} \mathbf{~ I})$ \\
\cline { 2 - 2 } & $\mathbf{n}$ (\%) \\
\hline Total number of patients with TEAEs & $429(82.3)$ \\
Injection site pain & $97(18.6)$ \\
Insomnia & $79(15.2)$ \\
Akathisia & $70(13.4)$ \\
Headache & $59(11.3)$ \\
Abnormal weight gain & $51(9.8)$ \\
Upper respiratory tract infection & $46(8.8)$ \\
Dizziness & $37(7.1)$ \\
Psychotic disorder & $42(8.1)$ \\
Weight increased & $39(7.5)$ \\
Nausea & $34(6.5)$ \\
Tremor & $33(6.3)$ \\
Worsening of schizophrenia & $33(6.3)$ \\
Anxiety & $29(5.6)$ \\
Somnolence & $27(5.2)$ \\
Restlessness & $26(5.0)$ \\
\hline
\end{tabular}

Abbreviation: TEAEs, treatment-emergent adverse events.

controlled studies (RCT) found similar outcomes after LAI and oral antipsychotic treatment. ${ }^{57}$ These results are at variance with the same group's earlier report indicating the superiority of LAIs versus oral antipsychotics in mirror-image comparison studies. ${ }^{58}$ The disparity of results from these two meta-analyses may be explained by patients in RCTs having better treatment adherence and a lesser severity of disease than real-world patients, whereas mirror-image studies better approximate real clinical practice. However, very few studies have assessed the impact of PP on hospitalizations..$^{52,55} \mathrm{We}$ used a mirror-design analysis to compare the "before" and "after" outcomes associated with PP on hospitalizations and days spent in hospital. In this 18-month open-label study, patients with recent-onset schizophrenia switched to PP had significantly fewer hospitalization days/person/year, decreasing from retrospective to the end of prospective study period (mean change from 74.3 to $18.9, P<0.0001$ ). Such reductions in hospital stay were also evident among patients with more than 1 year schizophrenia history (63.3 vs $19.2, P<0.0001)$ and in those who had been hospitalized in the past 1 year (186.9 vs 34.2, $P<0.0001)$. For sensitivity analysis, approximately $36 \%$ patients having $<1$ year schizophrenia history were excluded to prevent exaggeration of the duration of hospital stay as it is expressed as per person per year. Consistent with these findings, the proportion of patients hospitalized dropped significantly from $39.7 \%$ to $25 \%(P<0.001)$ over 18-month prospective period; and there was a rise in proportion of patients without hospitalization
( $60.3 \%$ vs $75 \%, P<0.001$ each), thereby indicating relatively more patients were "hospital-free" by the end of treatment. A similar trend was also seen among patients with $>1$ year history of schizophrenia ( $69.2 \%$ vs $75.5 \%, P<0.001$ each). These outcomes are consistent with the beneficial effect of LAIs found in the meta-analysis of Kishimoto et a ${ }^{56,57}$ in significantly reducing the number of hospitalizations in patients with schizophrenia, and support the use of PP in patients with recent-onset schizophrenia from the Asia-Pacific.

The safety profile of PP shown in this study was consistent with evidence on its tolerability and safety published in a number of short-term and long-term studies conducted in both Western and Asian populations. ${ }^{1,17,30-37,40,42}$ Of note, cardiac-related abnormalities were infrequent $(\leq 0.8 \%)$; glucose-related adverse events were also relatively very low $(0.6 \%)$ compared with $2.8 \%$ from a previous tolerability study in patients with recently diagnosed schizophrenia. ${ }^{17}$ The metabolic profile of PP observed in our study is consistent to that outlined previously. ${ }^{59}$ However, $41.8 \%$ of the study population had clinically significant weight gain and this will remain an area needing careful monitoring. The incidence of potentially prolactin-related adverse effects ranged from $2.1 \%$ (sexual side effects in men) to $11.1 \%$ (amenorrhea) which is consistent with past findings in this subgroup of patients. ${ }^{17,32}$

EPS-related events are of particular concern in patients early in the course of illness, because they may have more susceptibility to adverse events than patients with chronic schizophrenia. ${ }^{34}$ Pertinent to this, the occurrence of EPS events in our study was relatively high (31\%) compared with $23.3 \%$ from a recent double-blind, randomized study of $\mathrm{PP}$ in predominantly Asian patients, ${ }^{42}$ and earlier studies. ${ }^{35,38,39,45,46}$ This could be attributed to greater sensitivity in the patients from our study who were early in the course of illness. A range of movement disorders including akathisia, dystonia, and Parkinsonism are likely to occur with exposure to antipsychotic medication in the first few years of illness. ${ }^{60-63}$ In this study, akathisia was the most commonly reported movement disorder over the entire study period. Low rates of Parkinsonism were observed (2.1\%) compared with other studies (7\%-8\%) in recently diagnosed schizophrenia. ${ }^{17,32}$ Overall, no new safety signals or unexpected results were detected in the Asia-Pacific population. Taken together, these long-term findings complement earlier tolerability analyses of oncemonthly injectable PP, confirming that patients with recently diagnosed schizophrenia can be satisfactorily switched to PP from oral antipsychotic treatments.

Some limitations of this study that should be considered are that the study lacked a comparator group and 
was open-label. This increases risk of the observational bias of the researchers as well as the hope and expectations of the patients. Clinical improvement may be due, in part, to a reversion to the mean, or alternatively an improvement in the clinical condition with time. Further, the rigor and structure of a clinical trial can improve treatment adherence, while regular review may help with clinical response all biasing the results to the prospectively recorded arm. The mirrorimage design also limits the type of outcome variable that can be used as few services routinely and accurately record changes in psychopathology as measured by scales such as PANSS. However, the endpoint used in this phase of the study - hospitalization - is of great clinical relevance and clearly signposted in past records. A mirror-image design can also be biased by a systematic change in policy over one period of such study, however, due to the diversity of countries across the Asian-Pacific region this is unlikely to have occurred. A further weakness of this part of the study is that the patients were treated with a nonstandardized range of medications during the retrospective phase of the study, making the comparison more difficult.

\section{Conclusion}

Treatment switch to PP from prior unsatisfactory oral antipsychotic therapy was effective and generally tolerable for patients (from the Asia-Pacific) with recent-onset schizophrenia, with significant improvements observed in clinical symptomatology over the prospective period and a significant reduction in the number of hospitalizations and days spent in hospital when compared to retrospective period before initiation of PP treatment.

\section{Acknowledgments}

The authors thank Ashwini Patil, MS (SIRO Clinpharm Pvt Ltd) for medical writing assistance and Wendy P Battisti, PhD, CMPP (Janssen Research and Development, LLC) for additional editorial assistance in the preparation of this manuscript. The authors thank the study participants, without whom this study would not have been accomplished, as well as the following investigators for their participation in this study:

Australia: Drs Damodaran S; Jespersen S; Lakshmana R; Farnbach P; Hustig H; Kulkarni J; Liu D; Harris A; New Zealand: Miles W; People's Republic of China: Drs Zhang H; Wang G; Tan Q; Li H; Fan J; Zhang J; Hong Kong: Drs Lo, T L W; Ng, M K, R; Korea: Drs Kim C; Lee C; Jung H; Jung I; Cho S; Chee I; Lee S; Lee B; Han C; Yoon J; Malaysia: Drs Singh Gill J; Mohd Yasin MA; Jasmit Singh SS; Mohd Yusoff
R; Abu Bakar AK; Dalip MD; Abdul Aziz S; Tuan Hadi TS; Mohd Noor MA; Philippines: Drs Cardinez-Tan MM; Sta Ana-Ponio B; Taiwan: Drs Liu C; Su T; Hsieh M; Chiu N; Jang F; Tang T; Thailand: Drs Chantarasak V; Kittiwattanagul $\mathrm{K}$; Pansawat W.

Data from this study were presented as a poster at the 5 th International Conference on Schizophrenia, held in India, from September 21-23, 2012; ASPR congress, Australia December 4-6, 2013; International Mental Health Conference, Australia, August 25-27, 2014.

\section{Author contributions}

CYK, CFC, TS, FZ, and AH designed the study and were responsible for data collection. SA was the project statistician and oversaw data analysis and interpretation. FZ contributed to the ideas of data analysis. PJ was involved in data analysis. All authors met ICMJE criteria and those who fulfilled the criteria are listed as authors. All authors had access to the study data, provided direction and formal review of the manuscript, and made the final decision about where to publish these data.

\section{Disclosure}

This study was funded by Janssen-Cilag Asia-Pacific Medical Affairs. The sponsor also provided formal review of this manuscript. CYK received honoraria for lectures from Janssen Korea. FZ is an employee of Xian Janssen Pharmaceuticals. CFC is an employee of Jan-Cil Singapore. SA is an employee of Janssen Research and Development, LLC. PJ is employed by Theorem Clinical Research and contracted to Xian Janssen Pharmaceuticals at the time of this analysis. Associate Professor Harris has received consultancy fees from Janssen Australia and Lundbeck Australia. He has received payments for educational sessions run for Janssen Australia and the Lundbeck Institute. He is or has recently been an investigator on industry-sponsored trials by Hoffman-La Roche, Janssen-Cilag Australia, and Brain Resource Ltd. Other authors report no other conflicts of interest.

\section{References}

1. Alphs L, Bossie CA, Sliwa JK, Fu DJ, Ma YW, Hulihan J. Paliperidone palmitate and risperidone long-acting injectable in subjects with schizophrenia recently treated with oral risperidone or other oral antipsychotics. Neuropsychiatr Disease Treat. 2013;9:341-350.

2. Kane JM, Garcia-Ribera C. Clinical guideline recommendations for antipsychotic long-acting injections. Br J Psychiatry Suppl. 2009; 52:S63-S67.

3. Morken G, Widen JH, Grawe RW. Non-adherence to antipsychotic medication, relapse and rehospitalisation in recent-onset schizophrenia. BMC Psychiatry. 2008;8:32. 
4. Nicholl D, Akhras KS, Diels J, Schadrack J. Burden of schizophrenia in recently diagnosed patients: healthcare utilisation and cost perspective. Curr Med Res Opin. 2010;26:943-955.

5. Schreiner A, Hargarter L, Hitschfield K, et al. Clinical effectiveness and resource utilization of paliperidone ER for schizophrenia: pharmacoepidemiologic international longitudinal antipsychotic registry (PILAR). Curr Med Res Opin. 2014;30:1279-1289.

6. Lacro JP, Dunn LB, Dolder CR, Leckband SG, Jeste DV. Prevalence of and risk factors for medication nonadherence in patients with schizophrenia: a comprehensive review of recent literature. J Clin Psychiatry. 2002;63:892-909.

7. Olivares JM, Pinal B, Cinos C. Comparison of long-acting antipsychotic injection and oral antipsychotics in schizophrenia. Neuropsychiatry. 2011;1:275-289.

8. Olivares JM, Thirunavukarasu M, Kulkarni J, Zhang HY, Zhang M, Zhang F. Psychiatrists' awareness of partial and nonadherence to antipsychotic medication in schizophrenia: results from an Asia-Pacific survey. Neuropsychiatr Disease Treat. 2013;9:1163.

9. Altamura AC, Aguglia E, Bassi M, et al. Rethinking the role of long-acting atypical antipsychotics in the community setting. Int Clin Psychopharmacol. 2012;27:336-349.

10. Valenstein M, Blow FC, Copeland LA, et al. Poor antipsychotic adherence among patients with schizophrenia: medication and patient factors Schizophrenia Bull. 2004;30:255-264.

11. Weiden PJ, Kozma C, Grogg A, Locklear J. Partial compliance and risk of rehospitalization among California medicaid patients with schizophrenia. Psychiatr Serv. 2004;55:886-891.

12. Robinson DG. Medication adherence and relapse in recent-onset psychosis. Am J Psychiatry. 2011;168:240-242.

13. Subotnik KL, Nuechterlein KH, Ventura J, et al. Risperidone nonadherence and return of positive symptoms in the early course of schizophrenia. Am J Psychiatry. 2011;168:286-292.

14. Bottlender R, Sato T, Jager M, et al. The impact of the duration of untreated psychosis prior to first psychiatric admission on the 15-year outcome in schizophrenia. Schizophrenia Res. 2003;62:37-44.

15. Harrigan SM, McGorry PD, Krstev H. Does treatment delay in firstepisode psychosis really matter? Psychol Med. 2003;33:97-110.

16. Emsley R, Oosthuizen P, Koen L, Niehaus DJ, Medori R, Rabinowitz J. Remission in patients with first-episode schizophrenia receiving assured antipsychotic medication: a study with risperidone long-acting injection. Int Clin Psychopharmacol. 2008;23:325-331.

17. Sliwa JK, Bossie CA, Fu DJ, Turkoz I, Alphs L. Long-term tolerability of once-monthly injectable paliperidone palmitate in subjects with recently diagnosed schizophrenia. Neuropsychiatr Disease Treat. 2012;8:375-385.

18. Robinson DG, Woerner MG, Delman HM, Kane JM. Pharmacological treatments for first-episode schizophrenia. Schizophrenia Bull. 2005;31:705-722.

19. Leucht C, Heres S, Kane JM, Kissling W, Davis JM, Leucht S. Oral versus depot antipsychotic drugs for schizophrenia - a critical systematic review and meta-analysis of randomised long-term trials. Schizophrenia Res. 2011;127:83-92.

20. Schreiner A, Aadamsoo K, Altamura AC, et al. A randomized, active-controlled rater-blinded 2-year study of paliperidone palmitate versus investigators' choice of oral antipsychotic monotherapy in patients with schizophrenia (PROSIPAL). 22nd European Congress of Psychiatry (European Psychiatric Association). Munich, Germany; 2014:188-189.

21. Alphs L, Mahalchick LC, Mao L, Starr L. Paliperidone research in demonstrating effectiveness (pride): functioning in subjects with schizophrenia and a history of incarceration. 167th Annual Meeting Of American Psychiatric Association. New York, USA; 2014.

22. Agid O, Foussias G, Remington G. Long-acting injectable antipsychotics in the treatment of schizophrenia: their role in relapse prevention. Expert Opin Pharmacother. 2010;11:2301-17.

23. Kane JM. Reducing non-adherence: new formulations of antipsychotic medications. Psychiatric Times. 2006;25.
24. Nasrallah HA. The case for long-acting antipsychotic agents in the post-CATIE era. Acta Psychiatr Scand. 2007;115:260-267.

25. Samtani MN, Sheehan JJ, Fu DJ, Remmerie B, Sliwa JK, Alphs L. Management of antipsychotic treatment discontinuation and interruptions using model-based simulations. Clin Pharmacol. 2012;4:25-40.

26. Schooler NR. Relapse and rehospitalization: comparing oral and depot antipsychotics. J Clin Psychiatry. 2003;64(Suppl 16):14-17.

27. Zhu B, Ascher-Svanum H, Shi L, Faries D, Montgomery W, Marder SR. Time to discontinuation of depot and oral first-generation antipsychotics in the usual care of schizophrenia. Psychiatr Serv. 2008;59:315-317.

28. Altamura AC, Sassella F, Santini A, Montresor C, Fumagalli S, Mundo E. Intramuscular preparations of antipsychotics: uses and relevance in clinical practice. Drugs. 2003;63:493-512.

29. Newton R, Hustig H, Lakshmana R, et al. Practical guidelines on the use of paliperidone palmitate in schizophrenia. Curr Med Res Opin. 2012;28:559-567.

30. Alphs L, Bossie CA, Sliwa JK, Ma YW, Turner N. Onset of efficacy with acute long-acting injectable paliperidone palmitate treatment in markedly to severely ill patients with schizophrenia: post hoc analysis of a randomized, double-blind clinical trial. Ann Gen Psychiatry. 2011;10:12

31. Attard A, Olofinjana O, Cornelius V, Curtis V, Taylor D. Paliperidone palmitate long-acting injection-prospective year-long follow-up of use in clinical practice. Acta Psychiatr Scandinavica. 2014;130:46-51.

32. Bossie CA, Fu DJ, Sliwa JK, Ma YW, Alphs L. Tolerability of initiation doses of once-monthly paliperidone palmitate in patients with recently diagnosed schizophrenia in an acute treatment trial. Ther Adv Psychopharmacol. 2011;1:111-124.

33. Coppola D, Liu Y, Gopal S, et al. A one-year prospective study of the safety, tolerability and pharmacokinetics of the highest available dose of paliperidone palmitate in patients with schizophrenia. BMC Psychiatry. 2012;12:26.

34. Fu DJ, Bossie CA, Sliwa JK, Ma YW, Alphs L. Paliperidone palmitate versus oral risperidone and risperidone long-acting injection in patients with recently diagnosed schizophrenia: a tolerability and efficacy comparison. Int Clin Psychopharmacol. 2014;29:45-55.

35. Gopal S, Vijapurkar U, Lim P, Morozova M, Eerdekens M, Hough D. A 52-week open-label study of the safety and tolerability of paliperidone palmitate in patients with schizophrenia. J Psychopharmacol. 2011;25:685-697.

36. Hough D, Gopal S, Vijapurkar U, Lim P, Morozova M, Eerdekens M. Paliperidone palmitate maintenance treatment in delaying the time-torelapse in patients with schizophrenia: a randomized, double-blind, placebo-controlled study. Schizophrenia Res. 2010;116:107-117.

37. Kramer M, Litman R, Hough D, et al. Paliperidone palmitate, a potential long-acting treatment for patients with schizophrenia. Results of a randomized, double-blind, placebo-controlled efficacy and safety study. Int J Neuropsychopharmacol. 2010;13:635-647.

38. Pandina G, Lane R, Gopal S, et al. A double-blind study of paliperidone palmitate and risperidone long-acting injectable in adults with schizophrenia. Prog Neuropsychopharmacol Biol Psychiatry. 2011;35:218-226.

39. Pandina GJ, Lindenmayer JP, Lull J, et al. A randomized, placebocontrolled study to assess the efficacy and safety of 3 doses of paliperidone palmitate in adults with acutely exacerbated schizophrenia. J Clin Psychopharmacol. 2010;30:235-244.

40. Taylor D, Olofinjana O. Long-acting paliperidone palmitate-interim results of an observational study of its effect on hospitalization. Int Clin Psychopharmacol. 2014;29:229-234.

41. Li H, Rui Q, Ning X, Xu H, Gu N. A comparative study of paliperidone palmitate and risperidone long-acting injectable therapy in schizophrenia. Prog Neuropsychopharmacol Biol Psychiatry. 2011; 35:1002-1008.

42. Takahashi N, Takahashi M, Saito T, et al. Randomized, placebocontrolled, double-blind study assessing the efficacy and safety of paliperidone palmitate in Asian patients with schizophrenia. Neuropsychiatr Disease Treat. 2013;8:1889-1898. 
43. Andreasen NC, Carpenter WT Jr, Kane JM, Lasser RA, Marder SR, Weinberger DR. Remission in schizophrenia: proposed criteria and rationale for consensus. Am J Psychiatry. 2005;162:441-449.

44. Fleischhacker W, Wolfgang RD, McQuade RN, Marcus DA, René S, William HC. A double-blind, randomized comparative study of aripiprazole and olanzapine in patients with schizophrenia. Biol Psychiatry. 2009;65:510-517.

45. Gopal S, Hough DW, Xu H, et al. Efficacy and safety of paliperidone palmitate in adult patients with acutely symptomatic schizophrenia: a randomized, double-blind, placebo-controlled, dose-response study. Int Clin Psychopharmacol. 2010;25:247-256.

46. Nasrallah HA, Gopal S, Gassmann-Mayer C, et al. A controlled, evidence-based trial of paliperidone palmitate, a long-acting injectable antipsychotic, in schizophrenia. Neuropsychopharmacology. 2010;35:2072-2082.

47. Weiden PJ, Schooler NR, Weedon JC, Elmouchtari A, Sunakawa A, Goldfinger SM. A randomized controlled trial of long-acting injectable risperidone vs continuation on oral atypical antipsychotics for first-episode schizophrenia patients: initial adherence outcome. J Clin Psychiatry. 2009;70:1397-1406.

48. Olivares JM, Rodriguez-Morales A, Diels J, et al. Long-term outcomes in patients with schizophrenia treated with risperidone long-acting injection or oral antipsychotics in Spain: results from the electronic schizophrenia treatment adherence registry (e-STAR). Eur Psychiatry. 2009;24:287-296.

49. Chue P, Llorca P-M, Duehesne I, Leal A, Rosillon D, Mehnert A. Hospitalization rates in patients during long-term treatment with longacting risperidone injection. J Appl Res. 2005;5(2):266-274.

50. Fuller M, Shermock K, Russo P, et al. Hospitalisation and resource utilisation in patients with schizophrenia following initiation of risperidone long-acting therapy in the veterans affairs healthcare system. J Med Econ. 2009;12:317-324.

51. Koczerginski D, Arshoff L. Hospital resource use by patients with schizophrenia: reduction after conversion from oral treatment to risperidone long-acting injection. Healthc Q. 2011;14:82-87.

52. Kozma CM, Slaton T, Dirani R, Fastenau J, Gopal S, Hough D. Changes in schizophrenia-related hospitalization and ER use among patients receiving paliperidone palmitate: results from a clinical trial with a 52-week open-label extension (OLE). Curr Med Res Opin. 2011;27:1603-1611.
53. Lambert T, Olivares JM, Peuskens J, et al. Effectiveness of injectable risperidone long-acting therapy for schizophrenia: data from the US, Spain, Australia, and Belgium. Ann Gen Psychiatry. 2011;10:10.

54. Su KP, Chang HC, Tsai SJ, Yen FC, Tang CH. Relapse and longacting injectable risperidone: a 1-year mirror image study with a national claims database in Taiwan. Value Health. 2009;12(Suppl 3): S118-S121.

55. Taylor M, Currie A, Lloyd K, Price M, Peperell K. Impact of risperidone long acting injection on resource utilization in psychiatric secondary care. J Psychopharmacol. 2008;22:128-131.

56. Willis M, Svensson M, Lothgren M, Eriksson B, Berntsson A, Persson U. The impact on schizophrenia-related hospital utilization and costs of switching to long-acting risperidone injections in Sweden. Eur J Health Econ. 2010;11:585-594.

57. Kishimoto T, Robenzadeh A, Leucht C, et al. Long-acting injectable vs oral antipsychotics for relapse prevention in schizophrenia: a metaanalysis of randomized trials. Schizophrenia Bull. 2014;40:192-213.

58. Kishimoto T, Nitta M, Borenstein M, Kane JM, Correll CU. Long-acting injectable versus oral antipsychotics in schizophrenia: a systematic review and meta-analysis of mirror-image studies. J Clin Psychiatry. 2013;74:957-965.

59. Gilday E, Nasrallah HA. Clinical pharmacology of paliperidone palmitate a parenteral long-acting formulation for the treatment of schizophrenia. Rev Recent Clin Trials. 2012;7:2-9.

60. Janno S, Holi M, Tuisku K, Wahlbeck K. Prevalence of neurolepticinduced movement disorders in chronic schizophrenia inpatients. Am J Psychiatry. 2004;161:160-163.

61. Kasper S. First-episode schizophrenia: the importance of early intervention and subjective tolerability. J Clin Psychiatry. 1999;60(Suppl 23): 5-9.

62. Kelly DL, Conley RR, Carpenter WT. First-episode schizophrenia: a focus on pharmacological treatment and safety considerations. Drugs. 2005;65:1113-1138.

63. Serretti A, De Ronchi D, Lorenzi C, Berardi D. New antipsychotics and schizophrenia: a review on efficacy and side effects. Curr Med Chem. 2004; 11:343-358.
Neuropsychiatric Disease and Treatment

\section{Publish your work in this journal}

Neuropsychiatric Disease and Treatment is an international, peerreviewed journal of clinical therapeutics and pharmacology focusing on concise rapid reporting of clinical or pre-clinical studies on a range of neuropsychiatric and neurological disorders. This journal is indexed on PubMed Central, the 'PsycINFO' database and CAS,

\section{Dovepress}

and is the official journal of The International Neuropsychiatric Association (INA). The manuscript management system is completely online and includes a very quick and fair peer-review system, which is all easy to use. Visit http://www.dovepress.com/testimonials.php to read real quotes from published authors. 